\title{
Evaluating the rate of contamination of raw milk with pathogenic biotypes of Yersinia enterocolitica in the North-East of Iran
}

\section{Fahimeh Movafagh}

Ferdowsi University of Mashhad

Tayebeh Zeinali ( $\sim$ ta.zeinaly@gmail.com )

Birjand University of Medical Sciences https://orcid.org/0000-0003-1596-1544

Abdollah Jamshidi

Ferdowsi University of Mashhad

\section{Research note}

Keywords: Yersinia enterocolitica; raw milk; biotype

Posted Date: July 10th, 2019

DOI: https://doi.org/10.21203/rs.2.11220/v1

License: (c) (i) This work is licensed under a Creative Commons Attribution 4.0 International License.

Read Full License 


\section{Abstract}

Objective: This study aimed to evaluate the contamination rate of raw milk to Y. enterocolitica and biotyping of isolated organisms. Results: The results showed 33\% of raw milk samples were contaminated with Yersinia enterocolitica. 1A biotype was the predominant one (26\%) but two pathogenic biotypes including 1B (6\%) and $5(1 \%)$ were also detected. Isolation of potentially pathogenic $\mathrm{Y}$. enterocolitica from raw milk and consequently its presence in dairy products which are made from raw milk is a public health hazard.

\section{Background}

Yersinia spp. as a member of enterobactericeae, consist of three species including Yersinia enterocolitica, Yersinia pseudotuberculosis and Yersinia pestis. Y. enterocolitica is a gram-negative bacillus ranging from coccobacilli to elongated bacilli, and some of them contain capsules. Yersinia enterocolitica is one of the most important food-borne pathogens which caused serious diseases in human [1]. Acute gastroenteritis is the major form of the disease caused by this organism but, mesenteric lymphadenitis mimicking appendicitis and septicemia are the other forms of the disease [1].

Yersinia is widely distributed in nature and has been detected in various kinds of food [2,3]. Y. enterocolitica is a psychrophilic organism and can replicate at refrigeration temperature. As such, the organism can survive in foods and liquids for a long period and raise a major public health problem [1].

Animal origin foods have a higher risk of gastrointestinal diseases caused by $Y$. enterocolitica in humans $[4,5]$. One of the most common consumed foods with animal origin is milk and dairy products. Furthermore, the presence of $Y$. enterocolitica in milk and dairy products were documented in several studies $[2,4,6]$.

Milk and dairy products are a major source of nutrients for humans [2]. Consumption of contaminated raw milk caused several outbreaks of gastrointestinal infections worldwide $[7,8]$. Consumption of raw milk is routine behavior in Iran, especially in rural areas. Some cases of diarrhea caused by $Y$. enterocolitica were documented in Iran [9].

European Food Safety Authority (EFSA) was recommended biotyping to determine the pathogenicity of isolated organisms [10] because of some biotypes of $Y$. enterocolitica including 1B, 2 and 5 are human and animal pathogens. Biotype $1 \mathrm{~A}$ is usually regarded as a non-pathogenic strain, although recently some virulence markers have been reported from the strains belonging to this biotype [11].

Moreover, presence of a large number of background microbiota, plus non-pathogenic Yersinia spp. and the low concentration of pathogenic strains, especially in food samples complicate the isolation that leads to an underestimated prevalence of pathogenic $Y$. enterocolitica in food [12]. Furthermore, biochemical confirmation of organism and biotyping need to use numerous biochemical tests and reagents [13]. Therefore, for the detection of $Y$. enterocolitica in food samples, PCR assays were designed. 
Molecular assays are rapid, highly specific and sensitive, and have been developed to overcome the limitations of conventional methods for the detection and identification of foodborne pathogens [13].

This is the first study that determined the prevalence of Y.enterocolitica in raw milk in the northeast of Iran and the presence of pathogenic strains. The aim of the present study was to evaluate the contamination rate of raw milk to $Y$. enterocolitica and biotyping of isolated organism.

\section{Main Text}

A total of 100 raw milk samples were obtained using stratified random sampling method from different milk collection centers (MCC) in northeast of Iran, from January 2017 until July 2017. Milk thoroughly mixed before sampling and then $50 \mathrm{ml}$ of milk was obtained in sterilized tubes and transport to the laboratory in the ice box, immediately. For enrichment, $10 \mathrm{ml}$ of each milk sample were aseptically transferred to $90 \mathrm{ml}$ of Peptone Sorbitol Broth (PSB) (Sigma-Aldrich, Germany) mixed for 5 min and incubated for 48 hours at $25^{\circ} \mathrm{C}$ in a shaker incubator. Amount of $0.5 \mathrm{ml}$ of each enriched sample mixed well with $4.5 \mathrm{ml}$ of $\mathrm{KOH} 0.25 \%$ and then streaked onto CIN agar plates (Merck, Darmstadt, Germany) supplemented with Cefsulodin-irgasan-novobiocin antibiotics (Merck, Darmstadt, Germany) [14]. Agar plates were incubated at $30^{\circ} \mathrm{C}$ for $24-48 \mathrm{~h}$. Small (1-2 mm diameter) colonies having deep red center with sharp border surrounded by clear colorless zone with entire edge in CIN agar plates were selected. Gram-negative bacilli with oxidase negative, catalase and urease-positive tests were presumptively regarded as Yersinia spp and selected for DNA extraction.

The DNA extracted from purified presumptive colonies using the boiling method [15]. PCR assay for amplification of $16 \mathrm{~s}$ rRNA was carried out in final volume of $20 \mu$, containing $1 \mu \mathrm{l}(10$ picomol) of forward (5'-AATACCGCATAACGTCTTCG-3') and reverse (5'-CTTCTTCTGCGAGTAACGTC-3') primer (Macrogen, South Korea), $2 \mu$ DNA template, $10 \mu$ of master mix (Ampliqon, Denmark) and $6 \mu \mathrm{l}$ Nuclease-free deionized distilled water. Thermal cycler program is presented in Table 1. Yersinia enterocolitica (ATCC 9610) and nuclease-free deionized water were used as the positive and negative control, respectively. PCR products were separated on a $1.5 \%$ agarose gel which was pre-stained by green viewer and photo documented under UV illumination.

For determination the biotype of isolates, esculin hydrolysis, indole production and lipase activity were investigated. Also, tests for fermentation of salicin, trehalose, sorbose and xylose were carried out [16].

According to the conventional culture method 35 out of 100 (35\%) raw milk samples were contaminated by Yersinia spp. Further, by molecular detection, 16s rRNA gene specific to Yersinia enterocolitica was amplified in 33 out of 35 contaminated samples. In other words, $33 \%$ of raw milk samples were detected to be contaminated by Yersinia enterocolitica in northeast of Iran. Figure 1 shows the amplification of the $16 \mathrm{~s}$ rRNA gene in raw milk samples. Among these 33 positive isolates, 26 isolates belonged to $1 \mathrm{~A}$ biotype (26\%), 6 isolates belonged to $1 \mathrm{~B}(6 \%)$ and 1 isolate belonged to $5(1 \%)$ biotypes. 
Milk contains various proteins, minerals and vitamins that made it a complete food especially for children as well as a rich medium for growth of microorganism. Therefore, hygienic quality and safety of this product is very important. One of the most prevalent health issues is food-borne diseases caused by a variety of bacterial, viral and parasitic micro-organisms. The most common cause of the bacterial foodborne disease is animal origin's food [17].

To our knowledge, such a high percentage of positive results (33\%) have never been reported in Iran. In another study, $1.6 \%$ of raw milk, but no pasteurized milk samples, were positive for $Y$. enterocolitica [5], which was lower than our results. Another study in Iran showed that $9.4 \%$ of milk and dairy products were contaminated with Yersinia spp. and 5.07\% were contaminated with $Y$. enterocolitica in culture method. Although in 16srRNA gene amplification, $4.34 \%$ of the samples were contaminated with this organism [18]. In another study, $Y$. enterocolitica was detected in 5.8\% of raw cow's milk [2]. These studies used different enrichment medium.

Alavi et al. [19] investigated the raw milk of sheep and goat regarding the presence of $Y$. enterocolitica. According to the reported results, 9 out of 84 sheep milk samples were contaminated with $Y$. enterocolitica in culture assay and all of the isolates confirmed through PCR assay, but the organism was not detected in 16 goat milk samples [19]. Hanifian and Khani [6] determined the prevalence of virulent Yersinia enterocolitica in raw milk and cheese samples. According to their results $7.62 \%$ of bulk raw milk samples were contaminated with $Y$. enterocolitica [6].

In Mexico City, Yersinia spp. was isolated from 454 out of 1300 raw cow's milk samples which were collected from stables. From the total isolated Yersinia genus, $44.25 \%$ were diagnosed as $Y$. enterocolitica [20]. In Turkey, $Y$. enterocolitica was the most common isolated species from raw milk; it was recovered from $47.3 \%$ of raw milk samples [21]. Alkali treatment was used in their isolation procedure prior to streaking on $\mathrm{CIN}$ agar. The reported rates of above studies are higher than the present study. According to a review, some studies suggested that alkali treatment may increase the rate of isolation of $Y$. enterocolitica but some others didn't propose it [13]. These observed variations in reported rate of contamination may be due to the isolation procedure. In the present study, enrichment in PSB and culture on CIN agar were used. Also, PCR confirmation of organism helps to rapid identification of $Y$. enterocolitica.

In the present study, most of the isolates (26 out of 33 ) belonged to $1 \mathrm{~A}$ biotype. The predominance of this biotype was also reported in the other studies $[2,5,21]$. Two pathogenic biotypes including $1 \mathrm{~B}$ and 5 , were isolated in the present study. Jamali et al. [2] reported that $15.8 \%$ of isolated $Y$. enterocolitica were belonged to $1 \mathrm{~B}$ biotype which was lower than the present study. Other studies were also isolated this biotype from animal and animal food origin [22,23]. Some researchers reported the other pathogenic biotypes in milk [20].

Contamination of raw milk with $Y$. enterocolitica is likely to be associated with inadequate hygienic practice in milking. Implementing of an effective sanitary milking management is the only way of controlling this pathogen at the farm level. The main sources of growth, propagation and survival of $Y$. 
enterocolitica are consumption of raw unpasteurized milk and use of dairy products produced from raw milk or with non-hygienic methods. Thus, sanitary milking, use of pasteurized milk for consumption and preparation of dairy products and protecting milk and dairy produce from dust and insects are the best ways to prevent $Y$. enterocolitica infections [18].

The conventional culture based method in detection of $Y$. enterocolitica is labor intensive, and require 3 to 4 days to reach the preliminary result. For confirmation, it needs more time and use of numerous phenotypic tests. Moreover, they need a sufficient number of organisms to distinguish the contamination while molecular confirmation method not only is very specific and sensitive but also is rapid and distinguish the viable cells in comparison with direct molecular test that can not differentiate the dead cells from viable ones [13].

In Conclusion, the recovery of potentially pathogenic biotypes of $Y$. enterocolitica from raw milk samples showed that consumption of raw milk and dairy products which are made from raw milk may impose a potential risk and raise a public health concern for food safety agencies. As detection of this pathogen is not performed routinely, further research on the prevalence of $Y$. enterocolitica in pasteurized milk and other foods with the animal origin is needed.

\section{Limitations}

One of the main limitations of this study is the lack of resources and funding to study the isolates with more detail.

\section{Declarations}

\section{Ethics approval and consent to participate}

All applicable international, national, and institutional guidelines for the care and use of samples were followed (Ferdowsi University of Mashhad, Code: 79).

\section{Consent to publish}

'Not applicable'

\section{Availability of data and material}

'Not applicable'

\section{Competing interests}

The authors declare that they have no competing interests.

\section{Funding}


This study was financially supported by vice chancellor in research affairs of veterinary faculty of Ferdowsi University of Mashhad.

\section{Authors' contributions}

TZ conceived and designed the study and, critically analyzed the data and wrote the manuscript. AJ coordinated and designed the study, critically analyzed the data and participated in the initial draft and the revision of the manuscript. FM Performed the experiment. All of the authors read and proved the final version of the manuscript.

\section{Acknowledgements}

Authors express their special thanks to Mrs Khajehnasiri for her technical assistance.

\section{References}

1. Bottone, E. J. (2015). Yersinia enterocolitica: Revisitation of an Enduring Human Pathogen. Clinical Microbiology Newsletter, 37(1), 1-8.

2. Jamali, H., Paydar, M., Radmehr, B., \& Ismail, S. (2015). Prevalence, characterization, and antimicrobial resistance of Yersinia species and Yersinia enterocolitica isolated from raw milk in farm bulk tanks. Journal of Dairy Science, 98(2), 798-803.

3. Zhang, H., Feng, S., Zhao, Y., Wang, S., \& Lub, X. (2015). Detection of Yersinia enterocolitica in milk powders by cross-priming amplification combined with immunoblotting analysis. International Journal of Food Microbiology, 214, 77-82.

4. Myers, K. M., Gaba, J., \& Al-Khaldi, S. F. (2006). Molecular identification of Yersinia enterocolitica isolated from pasteurized whole milk using DNA microarray chip hybridization. Molecular and Cellular Probes, 20(2), 71-80.

5. Soltan-Dallal, M.-M., Tabarraie, A., \& MoezArdalan, K. (2004). Comparison of four methods for isolation of Yersinia enterocolitica from raw and pasteurized milk from northern Iran. International Journal of Food Microbiology, 94, 87-91.

6. Hanifian, S., \& Khani, S. (2012). Prevalence of virulent Yersinia enterocolitica in bulk raw milk and retail cheese in northern-west of Iran. International Journal of Food Microbiology, 155, 89-92. doi:10.1016/j.ijfoodmicro.2012.01.012

7. Ackers, M.-L., S. Schoenfeld, J. Markman, M. G. Smith, M. A. Nicholson, W. DeWitt, . . Slutsker, L. (2000). An outbreak of Yersinia enterocolitica 0: 8 infections associated with pasteurized milk. Journal of Infectious Diseases, 181, 1834-1837.

8. Barrett, N. J. (1986). Communicable disease associated with milk and dairy products in England and Wales: 1983-1984. Journal of Infection, 12, :265-272.

9. Kebria, F. G., Khodabakhshi, B., Kouhsari, H., Sheshpoli, M. S., Behnampoor, N., Livani, S., . . Ghaemi, E. A. (2010). Yersinia Enterocolitica in Cases of Diarrhea in Gorgan, Northern Iran. Medical Laboratory 
Journal, 4(1).

10. EFSA, E. F. S. A. (2007). Opinion of the Scientific Panel on Biological Hazards on monitoring and identification of human enteropathogenic Yersinia spp. The EFSA Journal, 595, 1-30.

11. Huovinen, E., Sihvonen, L. M., Virtanen, M. J., Haukka, K., Siitonen, A., \& Kuusi, M. (2010). Symptoms and sources of Yersinia enterocolitica -infection: a case-control study. BMC Infect. Dis, 10, 122. doi:http://www.biomedcentral.com/1471-2334/10/122

12. Bonardi, S., Paris, A., Bassi, L., Salmi, F., Bacci, C., Riboldi, E., . . Brindani, F. (2010). Detection, semiquantitative enumeration, and antimicrobial susceptibility of Yersinia enterocolitica in pork and chicken meats in Italy. . J. Food Prot., 73, 1785-1792. doi:http://dx.doi.org/10.1016/j.jffoodmicro.2013.02.012.

13. Petsios, S., Fredriksson-Ahomaa, M., Sakkas, H., \& Papadopoulou, C. (2016). Conventional and molecular methods used in the detection and subtyping of Yersinia enterocolitica in food. International Journal of Food Microbiology, 237, 55-72.

14. Van Damme, I., Berkvens, D., Botteldoorn, N., et al. (2013). Evaluation of the ISO 10273:2003 method for the isolation of human pathogenic Yersinia enterocolitica from pig carcasses and minced meat. Food Microbiol; 36(2): 170-175.

15. Zeinali, T., Jamshidi, A., Rad, M., \& Bassami, M. (2015). A comparison analysis of Listeria monocytogenes isolates recovered from chicken carcasses and human by using RAPD PCR. Int $J$ Clin Exp Med, 8(6), 0152-10157.

16. Ye, Q., Wu, Q., Hu, H., Zhang, J. and Huang, H. (2016). Prevalence and characterization of Yersinia enterocolitica isolated from retail foods in China. Food Control; 61: 20-27.

17. World Health Organisation (WHO). (2007). Food Safety and Food-borne Illness.

18. Rahimi, E., Sepehri, S., Dehkordi, F. S., Shaygan, S., \& Momtaz, H. (2014). Prevalence of Yersinia Species in Traditional and Commercial Dairy Products in Isfahan Province, Iran. Jundishapur J Microbiol., 7(4), e9249.

19. Alavi, S. M., Rahimi, E., \& Tajbakhsh, E. (2017). Prevalence of Yersinia enterocolitica in raw small ruminant milk in shahrekord, iran. Bulgarian Journal of Veterinary Medicine, (online first), 7. doi:10.15547/bjvm.1088

20. Bernardino-Varo, L., Quinones-Ramirez, E. I., Fernandez, F. J., \& Vazquez-Salinas, C. (2013). Prevalence of Yersinia enterocolitica in raw cow's milk collected from stables of Mexico city. Journal of Food Protection, 76(4), 694-698. doi:10.4315/0362-028X.JFP-12-325

21. Yucel, N., \& Ulusoy, H. (2006). A Turkey survey of hygiene indicator bacteria and Yersinia enterocolitica in raw milk and cheese samples. Food Control, 17, 383-388.

22. Bonardi, S., Bruini, I., D'Incaub, M., Damme, I. V., Carniel, E., Brémont, S., . . Brindani, F. (2016). Detection, seroprevalence and antimicrobial resistance of Yersinia enterocolitica and Yersinia pseudotuberculosis in pig tonsils in Northern Italy. International Journal of Food Microbiology, 235, 125-132. 
23. Tan, L. K., Ooi, P. T., \& Thong, K. L. (2014). Prevalence of Yersinia enterocolitica from food and pigs in selected states of Malaysia. Food Control, 35, 94-100.

\section{Tables}

Table 1. Thermal cycler program of amplification of 16s rRNA gene of $Y$. enterocolitica

\begin{tabular}{|c|c|c|c|c|c|c|c|}
\hline Primer & $\begin{array}{l}\text { Initial } \\
\text { Denaturation }\end{array}$ & $\begin{array}{l}\text { Number } \\
\text { Cycles }\end{array}$ & of & Denaturation & Annealing & Extension & $\begin{array}{l}\text { Final } \\
\text { Extension }\end{array}$ \\
\hline $\begin{array}{l}16 s \\
\text { rRNA }\end{array}$ & $94^{\circ} \mathrm{C}: 5 \mathrm{~min}$ & 36 & & $94^{\circ} \mathrm{C}: 45 \mathrm{sec}$ & $\begin{array}{l}62^{\circ} \mathrm{C}: \\
\text { sec }\end{array}$ & $\begin{array}{l}72{ }^{\circ} \mathrm{C}: 45 \\
\text { sec }\end{array}$ & $72^{\circ} \mathrm{C}: 7 \mathrm{~min}$ \\
\hline
\end{tabular}

\section{Figures}

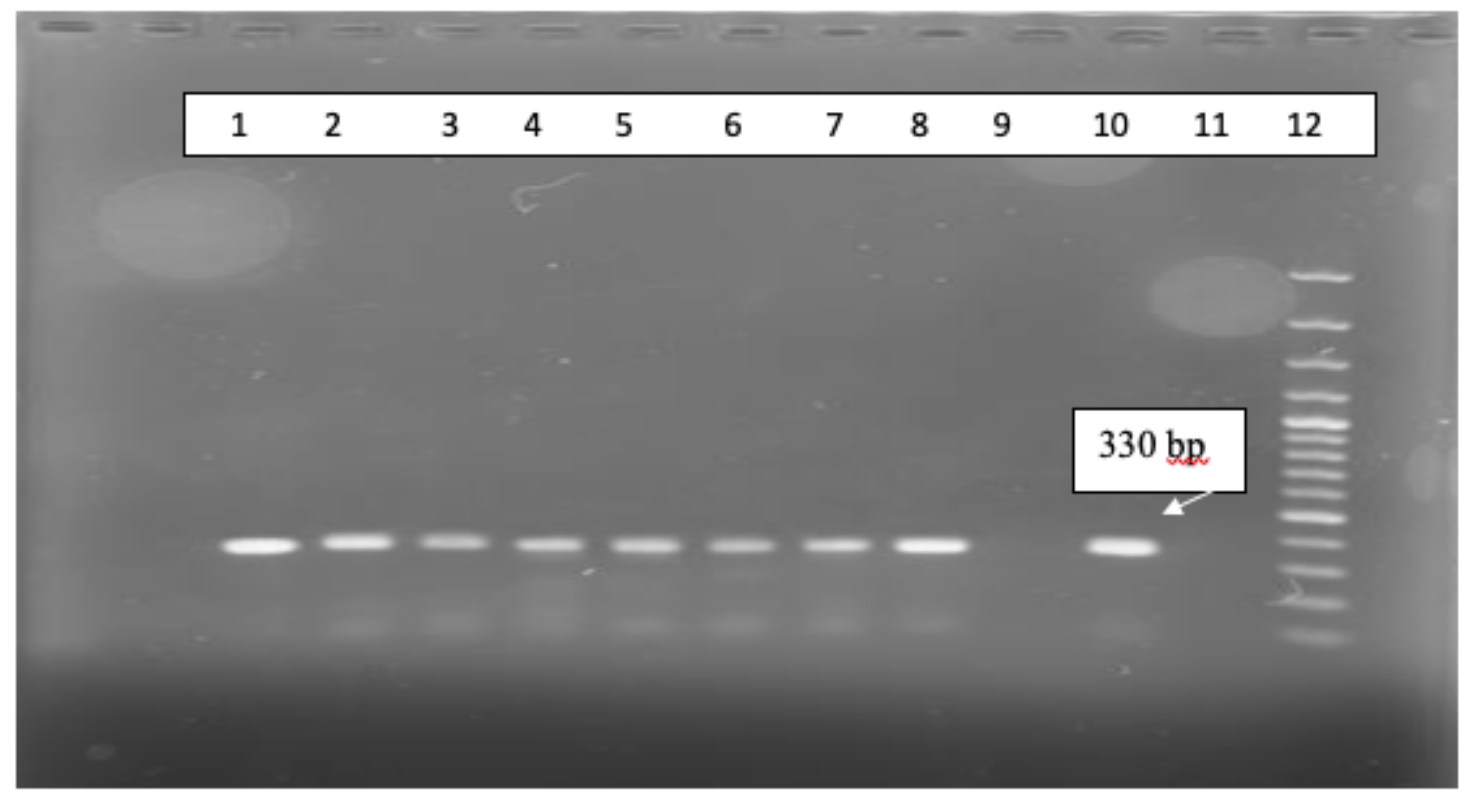

\section{Figure 1}

PCR amplification of 16s rRNA gene of Y. enterocolitica. Lane 1 to 8 positive sample; Lane 9: Negative Control; Lane 10: Positive Control; Lane 12: 100 base-pare plus DNA Ladder. 